\title{
ORIGINAL
}

\section{Association of social deprivation with 1-year outcome of ICU survivors: results from the FROG-ICU study}

\author{
Kathleen Bastian 1,2,3, Alexa Hollinger 1,2,3, Alexandre Mebazaa ${ }^{1,2,4}$, Elie Azoulay 1,4, Elodie Féliot ${ }^{1}$, \\ Karine Chevreul1,6, Marie-Céline Fournier ${ }^{1,2}$, Bertrand Guidet ${ }^{7}$, Morgane Michel ${ }^{6}$, Philippe Montravers ${ }^{4,8}$, \\ Sébastien Pili-Floury ${ }^{9,10}$, Romain Sonneville ${ }^{11}$, Martin Siegemund ${ }^{3}$ and Etienne Gayat ${ }^{1,2,4^{*}}$ (D) on behalf of the \\ FROG-ICU Study Investigators
}

(C) 2018 Springer-Verlag GmbH Germany, part of Springer Nature and ESICM

\begin{abstract}
Purpose: Intensive care unit survivors suffer from prolonged impairment, reduced quality of life, and higher mortality rates after discharge compared to the general population. Socioeconomic status may play a partial but important role in mortality and recovery. Therefore, the detection of factors that are responsible for poor long-term outcomes would be beneficial in designing targeted interventions for at-risk populations.

Methods: For an endpoint analysis, 1834 intensive care unit patients with known French Deprivation Index (FDep) scores were included from the French and euRopean Outcome reGistry in Intensive Care Units (FROG-ICU) study, which was a prospective, observational, multicenter cohort study performed in 20 French intensive care units in 13 different hospitals. Socioeconomic status was defined by using the FDep score [represented as quintiles when referring to the general French population, as quintiles when referring to the FROG-ICU cohort, or as dichotomized data (which was defined as a FDep $\leq 0$ for nondeprived patients)] and by using a detailed social questionnaire that was completed 3 months after discharge. The primary outcome included an all-cause, 1-year mortality after ICU discharge when regarding socioeconomic status. The secondary outcomes included both ICU and hospital lengths of stay, both short- and medium-term mortality, and the quality of life, as assessed during the 1-year follow-up by using the Medical Outcome Survey Short Form-36 (SF-36). The Revised Impact of Event Scale (IES-R) was used to evaluate the symptoms of post-traumatic stress disorder, and the Hospital Anxiety and Depression Scale (HADS) was used to screen for anxiety and depression.

Results: Of the 1447 patients who were discharged alive from the ICU, 19.2\% died over the following year. No association was found between 1-year mortality and socioeconomic status, regardless of whether this association was analyzed in quintiles ( $p=0.911$ in the quintiles of the general French population; $p=0.589$ in the quintiles of the FROG-ICU cohort itself) or as dichotomized data [nondeprived ( $n=177$; 1-year mortality of 18.2\%) versus deprived $(n=97 ; 1$-year mortality of 20.5\%; $p=0.304)]$. Moreover, no differences were found between the nondeprived and the
\end{abstract}

\footnotetext{
*Correspondence: etienne.gayat@aphp.fr

1 Department of Anaesthesiology and Reanimation, Burn and Critical Care Medicine, AP-HP, Saint Louis and Lariboisière University Hospitals, 2 Rue Ambroise Paré, 75010 Paris, France

Full author information is available at the end of the article

Kathleen Bastian and Alexa Hollinger contributed equally to study design and writing of the manuscript.
}

\section{实 Springer}


deprived patients in the ICU and hospital lengths of stay, ICU mortalities, in-hospital mortalities, or 28-day mortalities. The SF-36 was below the score for the normal French population throughout the follow-up period. Socially deprived patients showed significantly lower median scores in the physical function subscale [55, interquartile range (IQR) (28.8-80) vs. 65, IQR (35-90); $p=0.014]$, the physical role subscale [25, IQR (0-75) vs. 33.3, IQR (0-100); $p=0.022]$, and the overall physical component scale [47.5, IQR (30-68.8) vs. 54.4, IQR (35-78.8); $p=0.010]$. Up to $31.6 \%$ of survivors presented symptoms that indicated post-traumatic stress disorder, and up to $31.5 \%$ of survivors reported clinically meaningful symptoms of anxiety or depression.

Conclusions: A lower socioeconomic status was associated with lower self-reported physical component scores in the nondeprived patients. Psychiatric symptoms are frequently reported after an ICU stay, and subsequent interventions should target those fields.

Trial registration: ClinicalTrials.gov NCT01367093; registered on June 6, 2011.

Keywords: Socioeconomic status, Post-traumatic stress disorder, FDep, IES-R, SF-36, HADS

\section{Background}

The burden that arises after intensive care treatment is significant: critical illness survivors can suffer longlasting physical, functional, and cognitive psychological impairments $[1,2]$. Disability and mortality after an intensive care unit (ICU) discharge remain high. Moitra et al. recently reported a 1-year mortality of $26.6 \%$ in an elderly ( $>65$ years old) cohort of 34,696 patients, ranging from $19.4 \%$ to $57.8 \%$, which was dependent on the ICU length of stay [3]. Only after a period of months to several years does the risk of death in this population return to the level of the general population [4]. Patients who are admitted because of trauma and cardiovascular diseases seem to readjust at the fastest rate, with normal population survival rates being observed immediately after ICU discharge [5].

A significant part of the variation in health is interconnected with socioeconomic status with regard to education, income, or geographical areas of residence and is independent of individual patient characteristics [6]. The assessment of the health-related quality of life of patients' has become more focused in recent decades. For example, a systematic review published in 2005 identified 21 studies that evaluated the quality of life in intensive care survivors [7]. In almost all European countries, groups of lower socioeconomic status and/or educational status experienced substantially higher mortality rates accompanied by lower scores in self-reported healthassessments (e.g., the patients with a higher socioeconomic status showed a greater functional recovery after a myocardial infarction) [8]. In a recently published letter, no association between socioeconomic status and ICU mortality was found in one ICU from a high poverty rate territory [9]. Hence, the role of the socioeconomic burden on mortality and convalescence after a critical illness remains poorly understood. The effects of all levels of socioeconomic status on the health and recovery of individuals need to be further investigated to allow target interventions for both ICU and post-ICU care [10].

Apart from the severities of underlying diseases, preadmission health statuses and comorbidities, treatments during ICU stays, and psychological and behavioral aspects, we sought to evaluate the effects of socioeconomic status on mortality and health-related quality of life after surviving a critical illness by using the French Deprivation Index (FDep).

\section{Methods \\ Study design}

This report describes an ancillary study from the French and euRopean Outcome reGistry in Intensive Care Units (FROG-ICU) cohort. The FROG-ICU study was a prospective, observational, multicenter cohort study of 2087 patients and was designed to investigate the long-term mortality rates of critically ill adult patients. The study was performed in accordance with the Good Clinical Practice standards and the tenets of the Declaration of Helsinki 2002, was approved by the local ethical committees, and was registered on ClinicalTrials.gov (NCT01367093).

The study design was published previously [11]. Briefly, all patients admitted to any of the 28 participating ICUs in 19 hospitals in France and Belgium were screened for eligibility. The inclusion criteria included the use of invasive mechanical ventilation and/or treatment with a positive inotropic agent for more than $24 \mathrm{~h}$. The exclusion criteria included an age of less than 18 years, a severe head injury, brain death or a persistent vegetative state, pregnancy or breastfeeding, an organ transplantation that occurred in the past 12 months, and a lack of social security coverage.

Of the 2087 patients from the total FROG-ICU cohort, we included 1834 patients in whom the FDep was obtained. As a result of the substudy design, the Belgian 
centers were excluded, which left the populations of 20 ICUs in 13 different hospitals in France for analysis.

The primary endpoint was an all-cause, 1-year mortality after ICU discharge by socioeconomic status, which was defined in terms of the FDep at the time of study inclusion. For cross-evaluation, each surviving patient was asked to complete a detailed social questionnaire (Supplementary Material) 3 months after ICU discharge. Furthermore, ICU and hospital lengths of stay, ICU mortalities, in-hospital mortalities, and 28-day mortalities were evaluated. Other secondary outcomes included the health-related quality of life, which was represented as the composite of physical and mental recoveries during the 1-year follow-up period by using the Medical Outcome Survey Short Form-36 (SF-36) [12]. The Revised Impact of Event Scale (IES-R) [13-15] was used to evaluate the symptoms of post-traumatic stress disorder (PTSD), and the Hospital Anxiety and Depression Scale (HADS) [16] was used to screen for anxiety and depression. The SF-36, IES-R, and HADS questionnaires were administered at 3,6 , and 12 months after ICU discharge.

\section{Evaluation of socioeconomic status French Deprivation Index}

The FDep is a multidimensional, area-based index that measures socioeconomic differences by using four census-derived variables with two positive and two negative dimensions: the median income per consumption unit or household, the rate of inhabitants educated to the 2-year university level, the unemployment rate, and the rate of blue-collar workers and employees in the labor force [17]. During the application of the index, the patients were categorized into five quintiles for the general French population, into quintiles for the FROG cohort, or were dichotomized as being "deprived", with a FDep score of greater than 0 , or as being "nondeprived", with a FDep score of 0 or less. In addition to the FDep, the annual income and the educational level were recorded in a subset of patients who completed a social questionnaire 3 months after ICU discharge.

\section{Social questionnaire}

The social questionnaire used in our study contained 21 questions that focused on various aspects of socioeconomic status. The two questions that overlapped with the FDep dimensions (annual income and educational level) were extracted for cross-validation of the FDep itself. The patients were divided into "low" $(<8000-12,000 €$ per year, $n=84)$, "intermediate" $(12,000-50,000 €, n=127)$, and "high" ( $>50,000-\geq 100,000 €, n=32$ ) income groups. Regarding educational level, the patients were grouped into "low" (primary school), "intermediate" (high school/ undergraduate), and "high" (postgraduate) educational levels.

\section{Evaluation of health-related quality of life}

The health-related quality of life was assessed using the SF-36, which contained eight multidimensional items with a maximum score of 100 . A higher score indicated a better mental and physical function [12]. The physical component scores (physical function, bodily pain, general health, and role limitations due to impaired physical function) were aggregated into a physical health component score (PCS), while the mental health component scores (mental health, vitality, role limitations due to emotional problems, and social function) were combined into the mental health component score (MCS). Normative population scores were evaluated for several countries [18]. In our substudy, we referred to previously determined values for the French general population [19].

\section{Evaluation of psychological impact \\ Evaluation of post-traumatic stress disorder}

The IES-R, which is a widely used symptom scale covering three main symptom clusters (intrusion, avoidance, and hyperarousability), was used to evaluate the symptoms of PTSD after ICU discharge. A cohort-dependent (e.g., a substance abuser or a veteran) cutoff score for the probability of a diagnosis of PTSD can be found in the literature and ranges from 22 to 44 points [13-15]. In our cohort, a score of 22 points was the threshold for a probable diagnosis of PTSD.

\section{Evaluation of anxiety and depression}

The HADS was used to screen for anxiety and depression. The scale contained 14 items in two subscales ("anxiety" and "depression"), with a higher score indicating greater distress. In our cohort, a score of at least 8 points indicated probable anxiety and depression [16]. It had been developed to screen for mental disorders within nonpsychiatric hospitals. In addition, it has been validated for use in general study populations and can be used as a simplified "case finder". Nevertheless, its power to detect anxiety and depression is considered to be inferior to a structured diagnostic interview.

\section{Statistical analysis}

The results are expressed as medians and interquartile ranges, or counts and percentages, as appropriate. The continuous variables were compared using the MannWhitney test or the Kruskal-Wallis test, as appropriate. The categorical variables were compared using a Chisquared test if the numbers were greater than 5 , or with the Fisher's exact test if the numbers were not greater than 5 . 
The primary analysis evaluated survival at 1-year after ICU discharge and was evaluated according to the socioeconomic status represented by the FDep category. Covariate-adjusted Cox regression models were used to determine the association between social deprivation and 1-year mortality (i.e., age, gender, alcohol consumption, depression, an infection with the human immunodeficiency virus [HIV], a loss of autonomy, other psychiatric disorders, a cognitive dysfunction, chronic obstructive pulmonary disease [COPD], malignant tumors, a chronic liver disease, a prior stroke, a prior myocardial infarction, smoking, the Charlson score, the Simplified Acute Physiology Score [SAPS] II value at admission, and the Sequential Organ Failure Assessment [SOFA] score at admission). The danger of death was expressed as a hazard ratio (HR) with a 95\% confidence interval (CI). Missing values were not replaced (see tables).

Any $p$ values that were less than 0.05 were considered to show a significant difference. All statistical analyses were performed using $\mathrm{R}$ software version 3.1.1 or above (The "R" Foundation for Statistical Computing, Vienna, Austria).

\section{Results}

In this study, 1834 patients were included and eligible from the FROG-ICU cohort. The patient characteristics are presented in Table 1. Of the 1169 (63.7\%) patients alive 1 year after ICU discharge, and by using the FDep information (i.e., 100\% socioeconomic status data; Fig. 1), we obtained complete information on the health-related quality of life in $566(48.4 \%)$ patients and complete information on psychological impacts in 143 (12.2\%) patients (Fig. 1). The basic comparisons of patients who were and were not successfully followed up to 1 year after ICU discharge are provided in Supplementary Table 1.

\section{Social deprivation and short- and long-term survival}

Among the 1834 patients with available FDep scores, 1228 patients (67\%) were categorized as being "nondeprived" and 606 patients were categorized as being "deprived". The patient characteristics are outlined in Table 1. There were no significant differences in either ICU lengths of stay or ICU mortalities (Table 1). Among quintiles of the FROG-ICU cohort and the general French population, concordance of $36 \%$ was found (Supplementary Table 2).

Among the 1834 patients with available FDep scores, 1447 (78.9\%) patients were discharged alive from the ICU. In those ICU survivors, analyses that were performed according to the quintiles of the FDep of the general French population showed an overrepresentation of patients with a high socioeconomic status (Supplementary Table 3). When the ICU survivors were dichotomized, $972(67.2 \%)$ were categorized as being "nondeprived" and 475 (32.8\%) were categorized as being "deprived".

No association was found between the 1-year mortality and socioeconomic status, regardless of whether the data were analyzed in the quintiles of the general French population, analyzed in the quintiles of the intensive care survivors of the FROG-ICU cohort $(p=0.911$, Table 2; $p=0.589$, Supplementary Table 4 ), or analyzed as being dichotomized (nondeprived: $n=177,1$-year mortality 18.2\%; deprived: $n=97,20.5 \%$; $p=0.304$; Table 1; Fig. 2; Supplementary Fig. 1). Moreover, we found no significant differences in any of the investigated outcomes (i.e., hospital lengths of stay, in-hospital mortalities, and 28-day mortalities; Table 1).

The FDep tends to decrease with a greater urbanization. Therefore, Paris and its suburbs are considered to be less deprived than rural areas. After excluding the central areas of Paris and the "Paris Urban Unit", only 322 patients remained for analysis. Even though this subgroup's median FDep was different [0.29, IQR (-3.1; $2.3)]$, the 1-year mortality remained unchanged (data not shown).

\section{Social deprivation and other secondary endpoints}

The health-related quality of life at 12 months after ICU discharge was still lower in our patients than in the normative data for the healthy French population (Fig. 3) [19]. However, there appeared to be a general increase in the health status scores over the year following ICU discharge (Fig. 3a, b). When the quintiles of the general French population were used for analysis, an inverse relationship between the FDep scores and the physical component scale scores at 1 year after discharge was observed, although this relationship was not statistically significant ( $p=0.108$; Table 2$)$. When the quintiles of the FROG cohort were applied, patients with a higher socioeconomic status had better physical component scale scores $(p=0.044$; Supplementary Table 4). Likewise, when the dichotomized representation was used for analysis, the nondeprived patients had better physical component scale scores [47.5 (30-68.8) vs. 54.5 (35-78.8); $p=0.010$; Fig. 4a, b; Table 3] than the deprived patients, while the trajectory of the mental component scale was similar in both groups.

No differences were observed between the nondeprived and deprived ICU survivors for the worst values of the HADS or the IES-R at 1 year after discharge (Table 3 ). Of note, there were lower response rates to the HADS (40.6\% of missing data, $n=588)$ and to the IES-R $(52.3 \%$ of missing data, $n=757$ ) questionnaires.

With a cutoff of 22 points, 149 patients from the nondeprived cohort (31.6\%) and 70 patients from the 
Table 1 Patient characteristics and outcome parameters categorized by French Deprivation Index (FDep)

\begin{tabular}{|c|c|c|c|c|}
\hline Patient characteristics & All patients $(n=1834)$ & FDep nondeprived ( $n=1228)$ & FDep deprived $(n=606)$ & $p$ value* \\
\hline FDep & $-0.6[-1.6 ; 0.3]$ & $-1.1[-2.1 ;-0.6]$ & $0.7[0.3 ; 1.1]$ & $<0.001$ \\
\hline \multicolumn{5}{|l|}{ Epidemiological data } \\
\hline Age (years) & $63[50 ; 74]$ & $63[51 ; 75]$ & $62[49 ; 72]$ & 0.024 \\
\hline Male gender (\%) & $1190(64.9 \%)$ & $794(64.7 \%)$ & $396(65.3 \%)$ & 0.771 \\
\hline Body mass index $\left(\mathrm{kg} / \mathrm{m}^{2}\right)$ & $26.4[23.1 ; 30.7]$ & $26.1[23 ; 30.5]$ & $27.2[23.5 ; 31.1]$ & 0.016 \\
\hline \multicolumn{5}{|l|}{ Past medical history, no. (\%) } \\
\hline Charlson score & $3[1,5]$ & $3[1,5]$ & $3[1,5]$ & 0.431 \\
\hline Alcohol abuse & $319(17.4 \%)$ & $214(17.4 \%)$ & $105(17.4 \%)$ & 0.976 \\
\hline Depression & $233(12.7 \%)$ & $165(13.4 \%)$ & $68(11.3 \%)$ & 0.186 \\
\hline HIV & $46(2.5 \%)$ & $36(2.9 \%)$ & $10(1.7 \%)$ & 0.100 \\
\hline Loss of autonomy & $70(3.8 \%)$ & $52(4.2 \%)$ & $18(3 \%)$ & 0.187 \\
\hline Other psychiatric disorder & $80(4.4 \%)$ & $61(5 \%)$ & $19(3.1 \%)$ & 0.072 \\
\hline Cognitive dysfunction & $31(1.7 \%)$ & $22(1.8 \%)$ & $9(1.5 \%)$ & 0.637 \\
\hline COPD & $240(13.1 \%)$ & $147(12 \%)$ & $93(15.4 \%)$ & 0.042 \\
\hline Active malignant tumor & $246(13.4 \%)$ & $157(12.8 \%)$ & $89(14.7 \%)$ & 0.253 \\
\hline Chronic liver disease & $140(7.6 \%)$ & $90(7.3 \%)$ & $50(8.3 \%)$ & 0.475 \\
\hline Prior stroke & $69(3.8 \%)$ & $55(4.5 \%)$ & $14(2.3 \%)$ & 0.022 \\
\hline Prior myocardial infarction & $68(3.7 \%)$ & 47 (3.8\%) & $21(3.5 \%)$ & 0.707 \\
\hline Smoking & $489(26.7 \%)$ & $335(27.3 \%)$ & $154(25.5 \%)$ & 0.412 \\
\hline ICU admission diagnosis, no. (\%) & & & & 0.006 \\
\hline Septic shock & 419 (22.9\%) & $269(21.9 \%)$ & $150(24.8 \%)$ & \\
\hline Acute respiratory failure & $352(19.2 \%)$ & $232(18.9 \%)$ & $120(19.8 \%)$ & \\
\hline Acute neurological disorder & $256(14 \%)$ & $184(15 \%)$ & $72(11.9 \%)$ & \\
\hline Out-of-hospital cardiac arrest & $161(8.8 \%)$ & $125(10.2 \%)$ & $36(5.9 \%)$ & \\
\hline Trauma & $86(4.7 \%)$ & $50(4.1 \%)$ & $36(5.9 \%)$ & \\
\hline \multicolumn{5}{|l|}{ Organ dysfunction scores } \\
\hline SAPS II score at admission & $48[35 ; 62]$ & $49[36 ; 63]$ & $46[35 ; 61]$ & 0.084 \\
\hline SOFA score at admission & $7[5,10]$ & $8[5,10]$ & $7[5,11]$ & 0.974 \\
\hline \multicolumn{5}{|l|}{ Status at admission } \\
\hline SBP & 122 [109; 139] & $122[109 ; 139]$ & $123[108 ; 140]$ & 0.854 \\
\hline DBP & $60[53 ; 70]$ & $61[53 ; 70]$ & $60[53 ; 70]$ & 0.826 \\
\hline $\mathrm{HR}$ & $90[77 ; 105]$ & $90[77 ; 105]$ & $90[78 ; 105]$ & 0.921 \\
\hline Creatinine & $82.8[58.8 ; 147]$ & $85[60 ; 156]$ & $79[57 ; 134]$ & 0.030 \\
\hline eGFR & $78.5[40.9 ; 119.4]$ & $76.3[38.1 ; 116.6]$ & $84.5[45.9 ; 126.8]$ & 0.023 \\
\hline Lactate & $1.4[1 ; 1.9]$ & $1.4[1 ; 1.9]$ & $1.5[1.1 ; 2.1]$ & 0.01 \\
\hline WBC count (g/l) & $10.8[7.5 ; 16.0]$ & $10.9[7.7 ; 16.0]$ & $10.7[7.3 ; 16.3]$ & 0.909 \\
\hline Hemoglobin & $9.9[8.9 ; 11.4]$ & $9.9[8.9 ; 11.4]$ & $10[8.9 ; 11.5]$ & 0.991 \\
\hline Platelet count (g/l) & $166[101 ; 247]$ & $167[102 ; 248]$ & $159[95.0 ; 244]$ & 0.237 \\
\hline \multicolumn{5}{|l|}{ Organ support/in ICU management } \\
\hline Mechanical ventilation & $1714(93.5 \%)$ & $1149(93.6 \%)$ & $565(93.2 \%)$ & 0.787 \\
\hline Tracheotomy & $284(15.5 \%)$ & $182(14.8 \%)$ & $102(16.8 \%)$ & 0.263 \\
\hline FFP transfusion (\%) & $326(17.8 \%)$ & $180(14.7 \%)$ & $146(24.1 \%)$ & $<0.001$ \\
\hline Inotrope/vasopressor (\%) & $1405(76.6 \%)$ & $931(75.8 \%)$ & $474(78.2 \%)$ & 0.253 \\
\hline RBC transfusion (\%) & $848(46.2 \%)$ & $535(43.6 \%)$ & $313(51.7 \%)$ & 0.001 \\
\hline RRT (\%) & $435(23.7 \%)$ & $289(23.5 \%)$ & $146(24.1 \%)$ & 0.792 \\
\hline Vasopressor (\%) & $120(6.5 \%)$ & $79(6.4 \%)$ & $41(6.8 \%)$ & 0.787 \\
\hline \multicolumn{5}{|l|}{ Status at discharge } \\
\hline SBP & 123 [109; 138] & $122[108 ; 138]$ & $124[110 ; 140]$ & 0.247 \\
\hline DBP & $67[57 ; 76]$ & $67[57 ; 75]$ & $66[56 ; 77]$ & 0.994 \\
\hline
\end{tabular}


Table 1 (continued)

\begin{tabular}{|c|c|c|c|c|}
\hline Patient characteristics & All patients $(n=1834)$ & FDep nondeprived $(n=1228)$ & FDep deprived $(n=606)$ & $p$ value $^{*}$ \\
\hline$H R$ & $89[78 ; 101]$ & $90[78 ; 101]$ & $89[78 ; 100.8]$ & 0.793 \\
\hline Creatinine & $68[52 ; 103.2]$ & $69[52.4 ; 105]$ & $67[51 ; 100]$ & 0.151 \\
\hline eGFR & $99.2[60.2 ; 139.4]$ & $98.1[58.6 ; 137.5]$ & $102.3[62.6 ; 142]$ & 0.087 \\
\hline Lactate & $1.4[1 ; 2.1]$ & $1.4[1 ; 2.1]$ & $1.4[1 ; 2.1]$ & 0.581 \\
\hline WBC count (g/l) & $9.8[7.2 ; 13.9]$ & $9.7[7.2 ; 13.7]$ & $10[7.1 ; 14.0]$ & 0.387 \\
\hline Hemoglobin & $9.9[8.8 ; 11.1]$ & $9.9[8.8 ; 11.1]$ & $9.8[8.8 ; 11]$ & 0.813 \\
\hline Platelet count (g/l) & $275[169 ; 425]$ & $280[169 ; 428]$ & $270[170 ; 422]$ & 0.618 \\
\hline \multicolumn{5}{|l|}{ Outcome parameters } \\
\hline ICU LOS (days) & $13[7,22]$ & $12[7,21]$ & $14[8,22]$ & 0.097 \\
\hline Hospital LOS (days) & $23[12 ; 40]$ & $23[12 ; 40]$ & $24[13 ; 40]$ & 0.19 \\
\hline ICU mortality (\%) & $387(21.1 \%)$ & $256(20.8 \%)$ & $131(21.6 \%)$ & 0.704 \\
\hline In-hospital mortality (\%) & $490(26.7 \%)$ & $320(26.1 \%)$ & $170(28.1 \%)$ & 0.364 \\
\hline 28-day mortality (\%) ${ }^{\mathrm{a}}$ & $117(8.1 \%)$ & $72(7.4 \%)$ & $45(9.5 \%)$ & 0.172 \\
\hline 1 -year mortality $(\%)^{\mathrm{a}}$ & $274(19 \%)$ & $177(18.2 \%)$ & 97 (20.5\%) & 0.304 \\
\hline
\end{tabular}

COPD chronic obstructive pulmonary disease, DBP diastolic blood pressure, eGFR estimated glomerular filtration rate, FDep French Deprivation Index, FFP fresh frozen plasma, HIV human immunodeficiency virus, HR heart rate, ICU intensive care unit, LOS length of stay, $R B C$ red blood cell, $R R T$ renal replacement therapy, SAPS Simplified Acute Physiology Score, SBP systolic blood pressure, SOFA Sepsis-related Organ Failure Assessment, WBC white blood cell

* $p$ value from Chi-squared test or Mann-Whitney test

a Among ICU survivors

deprived cohort (14.7\%) were identified as having meaningful symptoms of PTSD. The difference between the groups was not statistically significant.

\section{Income and educational level and outcome parameters}

Self-reported data on annual financial resources were obtained from 312 (17\%) patients, and data on educational level were obtained from 289 (16\%) patients.

The cross-match analysis of annual income and educational level is presented in Supplementary Table 5 . When FDep was applied to the earnings groups, only $25 \%(n=21)$ of the patients from the low annual income group could be categorized as "deprived", with the remaining $75 \%(n=63)$ still categorized as "nondeprived" (Table 4). Likewise, 28.1\% $(n=9)$ of the patients in the high-income group could still be considered "deprived" (vs. "nondeprived": 71.9\%, $n=23$ ) (Table 4). A subgroup mortality analysis was not possible, as only two of these 312 patients died within 1 year after ICU discharge.

Among 243 patients with available information, the patients with low annual earnings were discharged from the ICU significantly earlier [median of 9, IQR (5-15.2) vs. 13 , IQR $(7-21.5)$ vs. 16 , IQR $(8,22)$ days; $p=0.011$; Table 4]. This was not true, however, when patients were categorized by educational level. Hospital lengths of stay were comparable among the annual income and educational level groups (Table 4).

The mental component scale and the physical component scale, both measured at 12 months, were positively associated with the level of annual reported income (as was the worst value during the post-ICU year) (Table 4). PCS but not MCS was associated with the level of education (Table 4).

Regarding psychological impact, a tendency towards higher HADS-Anxiety subscale scores and IES-R scores could be discerned for the low annual income group (data not shown).

\section{Discussion}

In this ancillary study of the FROG-ICU that enrolled the largest population of ICU patients at discharge, we observed no association between the social deprivation, as categorized by the FDep, and the outcome in critically ill patients. Overall, this finding could be considered a sign of quality of the French ICU standard operating procedures. However, whether the FDep can determine true differences in socioeconomic status has to be questioned. When the financial domain ("annual income") of the subgroup of patients who answered the social questionnaire was examined, and when they were classified as previously described, a significant difference between economic status and the outcome parameters was revealed. The mental component scale, the physical component scale, and the ICU and hospital lengths of stay vary considerably within the different income groups, and, to some extent, this effect applies to educational levels as well. When the FDep was applied to the previously classified income groups, as mentioned above, a possible contradictory classification may result (e.g., $n=63$ individuals considered to be "nondeprived", although they 


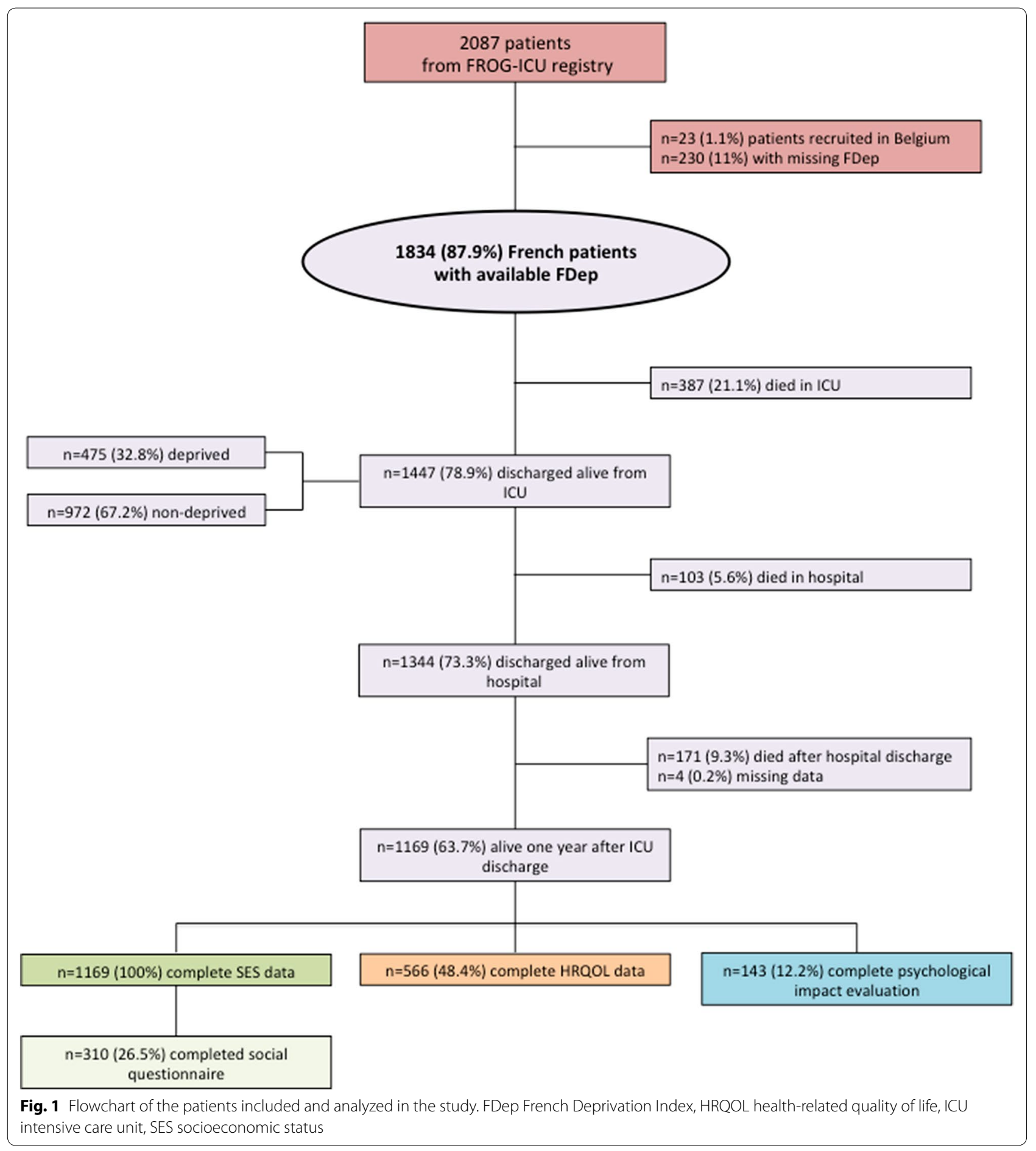

reported an income of $<8000-12,000 €$ per year). To truly define the impact of socioeconomic status on the healthrelated quality of life and mortality, without applying an easily accessible index, such as the FDep, a validation in a larger cohort, with a more specific investigation than the social questionnaire that we used, would be needed.
It must also be remembered that income and educational level are correlated.

It is somewhat surprising that no differences in mortality regarding socioeconomic status could be found in our cohort, which is contrary to previously reported data from several European and American studies. In 
Table 2 Outcomes in ICU survivors according to French Deprivation Index (FDep) quintiles of the general French population

\begin{tabular}{lllllll}
\hline Outcome & Q1 $(n=791)$ & Q2 $(n=230)$ & Q3 $(n=91)$ & Q4 $(n=152)$ & Q5 $(n=183)$ & $p$ value \\
\hline 1-year mortality & $147(18.6 \%)$ & $44(19.1 \%)$ & $15(16.5 \%)$ & $32(21.2 \%)$ & $36(19.8 \%)$ & $0.911[\mathrm{C}]$ \\
MCS at 12 months & $58.5[36.8 ; 77.9]$ & $65.7[40.6 ; 79.2]$ & $62.2[41.1 ; 77.9]$ & $55.9[37.2 ; 71.4]$ & $49.2[33.8 ; 72.6]$ & $0.27[\mathrm{k}]$ \\
PCS at 12 months & $55.6[35.5 ; 78.8]$ & $51.2[30 ; 71.9]$ & $45.6[28.8 ; 77.5]$ & $48.8[30.9 ; 68.8]$ & $45[30 ; 65]$ & $0.108[\mathrm{k}]$ \\
\hline
\end{tabular}

MCS mental health component score, PCS physical health component score, FDep French Deprivation Index, ICU intensive care unit, LOS length of stay, [c] Chi ${ }^{2}$ [ $\left.k\right]$ Kruskal-Wallis

FDep quintiles of the general French population:

FDep $\leq-0.38384$ : Quintile FDep $=1$

$-0.38384<$ FDep $\leq 0.12852$ : Quintile FDep $=2$

$0.12852<$ FDep $\leq 0.45389$ : Quintile FDep $=3$

$0.45389<$ FDep $\leq$ 0.77483: Quintile FDep $=4$

FDep $>0.77483$ : Quintile FDep $=5$

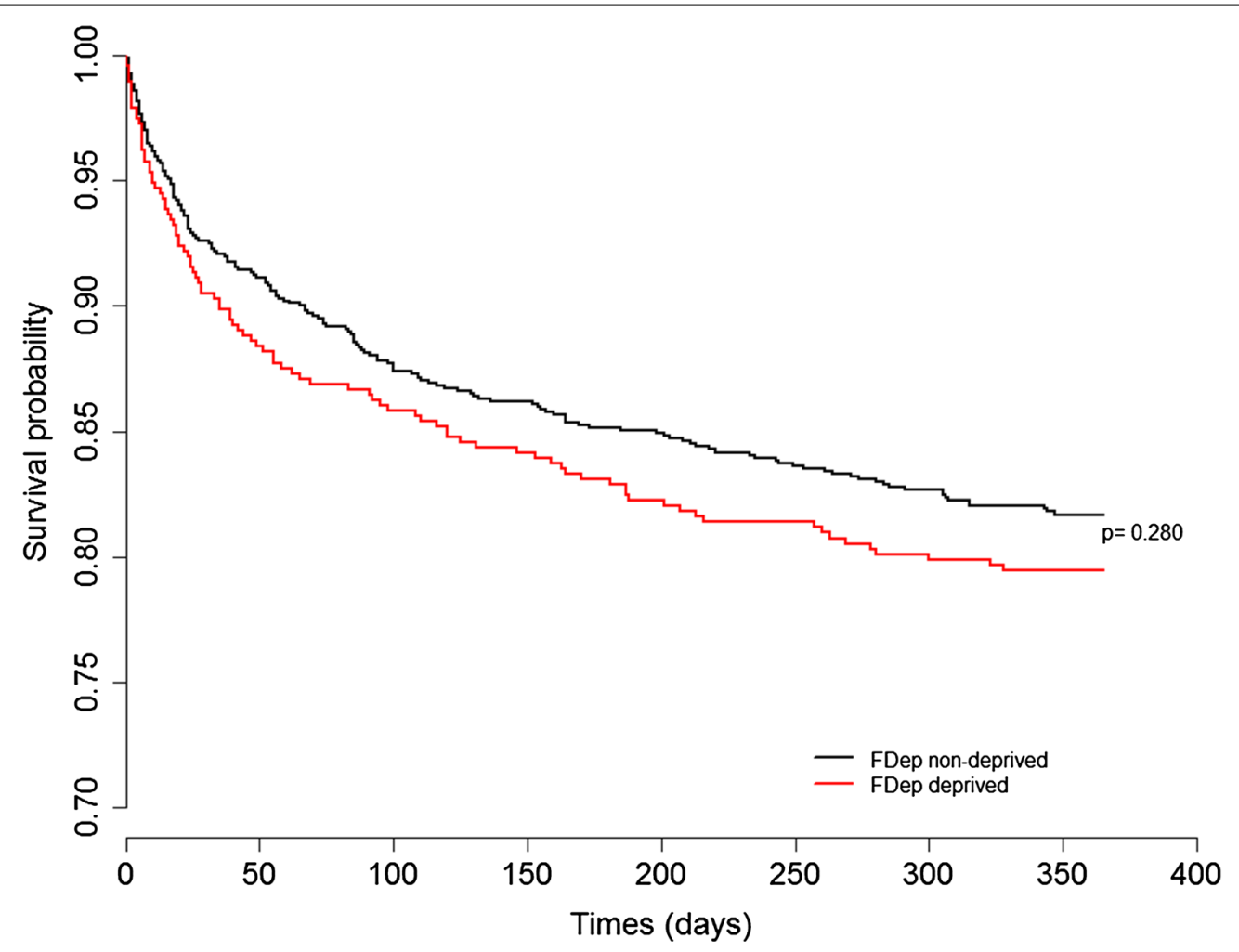

Fig. 2 Kaplan-Meier curve of survival at 1 year after ICU discharge according to FDep category

Europe, for instance, a German study, based on insurance data from 1987 to 1995 and including 416,000 patients, was able to show differences in mortality, depending on the professional status of the individuals. The study reported a four- to fivefold higher risk of long-term mortality for unskilled insured workers, compared to high-level executives [20]. When considering medical conditions, a social gradient in female breast cancer patients could be shown in a
Geneva-based study, regarding mortality and socioeconomic status with respect to occupation [21]. The associations of the risks of ICU admission with sepsis and socioeconomic status in critically ill patients were recently reported in a Danish study [22]. Furthermore, a very recent German study, focusing on surgical intensive care patients, described a longer duration of mechanical ventilation in patients with unfavorable socioeconomic characteristics, such as a high number 


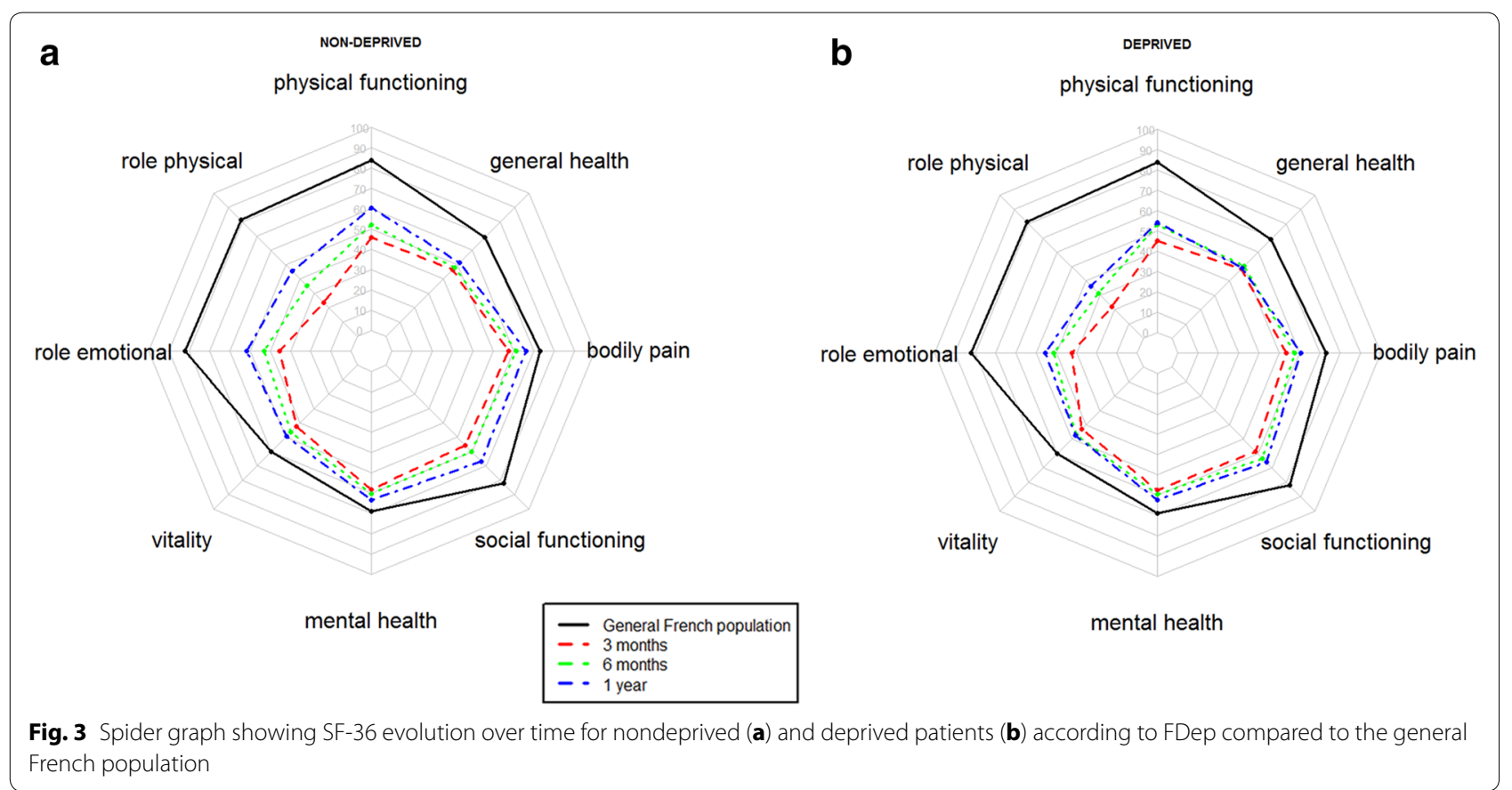

of people per household [23]. In the USA, when solely analyzing a geographically limited area, namely, Baltimore, and when dichotomizing the neighborhoods by median income level, the authors showed an association between lower household income communities and higher rates of death from sepsis [24]. Likewise, a nationwide cohort analysis study demonstrated that patients with sepsis who lived in the lowest median income quartile had a higher risk of mortality compared to residents of the highest income quartile after adjustment for the severity of illness [25]. This finding could be due to a late stage diagnosis in minority and low income groups, as was also reported in cancer patients in the USA [26]. Globally, when analyzing a large multinational study that covered 50 different countries, the authors found a high national income per capita in intensive care patients with acute respiratory distress syndrome (ARDS), with this high national income per capita also being associated with an increased survival [27]. As a result of our study design, the patients without insurance were excluded, and no further investigations regarding insurance status were made. However, excluding patients without health insurance might have created a selection bias and could also be one reason why no differences were found. Indeed, an Australian study found a reduced in-hospital mortality for ICU patients with a compensable status of insurance, although postdischarge ICU patients were not studied [28]. According to those studies, socioeconomic status influences outcomes in the critically ill, which is consistent with our results based on the social questionnaire. Hence, the deprivation index likely only focuses on a relevant portion of socioeconomic status.

Another aspect that could reduce the ability to show differences in outcomes among different socioeconomic status groups, as defined by the FDep, is the fact that the greatest portion of our study sample was derived from the Paris Urban Unit. As differences among outcome parameters for both groups remained unchanged, the FDep seems reliable in this context. However, this could be investigated in future studies by using other indexes (e.g., the French European Deprivation Index, FEDI).

The only statistically significant difference between the socially nondeprived and deprived survivors of critical illness was in the physical component scale at 1 year after ICU discharge. Likewise, with regard to financial resources, the low annual income group had significantly lower scores for the physical component scale and the mental component scale, with gradual increases observed with greater earnings. It remains unclear whether this difference was due to a lack of physical activity, a lack of support from cohabiting partners and family or limited access, or a lack of compliance, when it concerned followup consultations with the family doctor, or whether it is connected to the lower self-reported baseline quality of life in the socially deprived individuals. The 2017 OECD (Organisation for Economic Co-operation and Development) Health at a Glance [29] statistics emphasized the differences in health perception based on income, with $80 \%$ of "good" self-reported health being present in the 


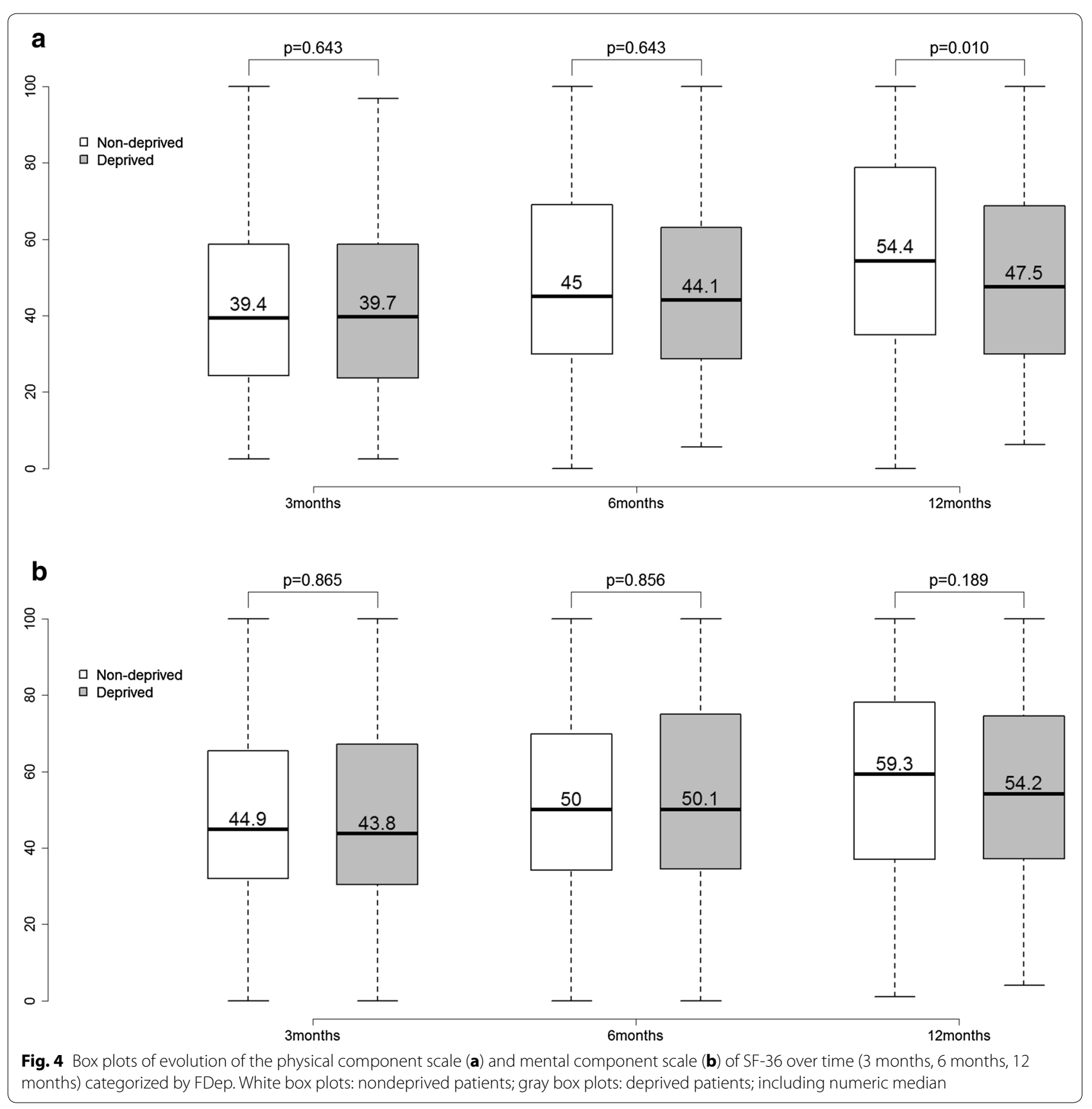

fifth quintile (the highest income group) and less than $60 \%$ being present in the lowest income group. Additionally, in all OECD countries (including France), except for New Zealand, Canada, and Australia, men were more likely to report being in good health [29]. Therefore, as a result of those variations, the overall perceived health status and self-reported SF-36 scores might differ to a certain extent.

The proportions of patients in our cohort with symptoms of anxiety (22.3-26.1\%), symptoms of depression
(18.7-30.4\%), and self-reported PTSD-like symptoms (9.5-15.1\%) after critical care are in concordance with previously reported data [30].

For the evaluation of the health-related quality of life, anxiety, and depression, we used a previously validated cutoff of 100 or less in the SF-36 and 8 or higher in the HADS and found no differences among the groups, which suggested no influence of socioeconomic status. However, for the IES-R, a wide range of known possible 
Table 3 Relation between socioeconomic status and health-related quality of life/psychological impact among ICU survivors

\begin{tabular}{|c|c|c|c|c|c|}
\hline & All patients, $n=1447$ & $\begin{array}{l}\text { FDep nondeprived, } \\
n=972\end{array}$ & FDep deprived, $n=475$ & $p$ value* & $\%$ of missing values \\
\hline HADS-A $\geq 8$ & $323(22.3 \%)$ & $218(22.4 \%)$ & $105(22.1 \%)$ & 0.899 & $588(40.6 \%)$ \\
\hline HADS-D $\geq 8$ & $270(18.7 \%)$ & $189(19.4 \%)$ & $81(17.1 \%)$ & 0.348 & $587(40.6 \%)$ \\
\hline IES-R $>22$ & $219(15.1 \%)$ & 149 (31.6\%) & $70(14.7 \%)$ & 0.931 & $757(52.3 \%)$ \\
\hline \multicolumn{6}{|c|}{ SF-36 3 months } \\
\hline MCS & 560 & $44.9[32.2 ; 65.3]$ & $43.8[30.5 ; 67.2]$ & 0.865 & 887 (61.3\%) \\
\hline PCS & 560 & $39.4[24.4 ; 58.8]$ & $39.7[23.8 ; 58.6]$ & 0.643 & 887 (61.3\%) \\
\hline \multicolumn{6}{|c|}{ SF-36 6 months } \\
\hline MCS & 556 & $50[34.2 ; 69.9]$ & $50.1[34.5 ; 74.7]$ & 0.856 & $891(61.6 \%)$ \\
\hline PCS & 559 & $45[30 ; 69.1]$ & $44.1[28.8 ; 63.1]$ & 0.643 & $888(61.4 \%)$ \\
\hline \multicolumn{6}{|c|}{ SF-36 12 months } \\
\hline MCS & 555 & $59.3[37 ; 78.1]$ & $54.2[37.2 ; 74.5]$ & 0.189 & $892(61.6 \%)$ \\
\hline PCS & 566 & $54.4[35 ; 78.8]$ & $47.5[30 ; 68.8]$ & 0.010 & $881(60.9 \%)$ \\
\hline
\end{tabular}

FDep French Deprivation Index, HADS Hospital Anxiety and Depression Scale, HADS-A anxiety subscale, HADS-D depression subscale, IES-R Impact of Event ScaleRevised, MCS mental component scale, PCS physical component scale, SES socioeconomic status, SF-36 Medical Outcome Survey Short Form-36

*From non-parametric Chi-squared test

Table 4 Subgroup analysis: social questionnaire regarding annual income and educational level with selected outcome parameters

\begin{tabular}{|c|c|c|c|c|c|}
\hline & Total population, $n=289$ & $\begin{array}{l}\text { Low educational } \\
\text { level }{ }^{\mathrm{a}}, n=64\end{array}$ & $\begin{array}{l}\text { Intermediate educa- } \\
\text { tional level }{ }^{\mathbf{b}}, n=114\end{array}$ & $\begin{array}{l}\text { High educational } \\
\text { levelc }^{c} n=111\end{array}$ & $p$ value \\
\hline FDep & $-0.6[-1.9 ; 0]$ & $-0.6[-1.6 ; 0]$ & $-0.4[-1.4 ; 0.5]$ & $-1.2[-2.2 ;-0.2]$ & $<0.001$ \\
\hline FDep $\leq 0$, nondeprived, no. (\%) & $214(74.0 \%)$ & $47(73.4 \%)$ & $73(64 \%)$ & $94(84.7 \%)$ & \\
\hline FDep >0, deprived, no. (\%) & 75 (26.0\%) & $17(26.6 \%)$ & $41(36 \%)$ & $17(15.3 \%)$ & \\
\hline MCS 12 months* & $59.7[37.4 ; 78.9]$ & $51.4[31.6 ; 72.9]$ & $58[35.5 ; 77]$ & $65.8[39.6 ; 81.2]$ & 0.087 \\
\hline PCS 12 months* & $55[33.4 ; 80]$ & $47.8[30.5 ; 66]$ & $54.4[33.8 ; 75.8]$ & $66.9[35 ; 86.2]$ & 0.035 \\
\hline ICU length of stay & $12[7,19]$ & $13[6.8 ; 23]$ & $11.5[7 ; 17.8]$ & $11[6,19]$ & 0.567 \\
\hline Hospital length of stay & $28.5[22.2 ; 45.8]$ & $27[16.8 ; 44.5]$ & $24[15 ; 39]$ & $22[12 ; 34]$ & 0.068 \\
\hline Low annual income, no. (\%) & $78(27.0 \%)$ & $27(56.2 \%)$ & $32(36 \%)$ & $19(20.2 \%)$ & $<0.001$ \\
\hline Intermediate annual income, no. (\%) & $121(41.9 \%)$ & $19(39.6 \%)$ & $53(59.6 \%)$ & $49(52.1 \%)$ & $<0.001$ \\
\hline \multirow[t]{2}{*}{ High annual income, no. (\%) } & $32(11.1 \%)$ & $2(4.2 \%)$ & $4(4.5 \%)$ & $26(27.7 \%)$ & $<0.001$ \\
\hline & Total population, $n=243$ & $\begin{array}{l}\text { Low annual } \\
\text { income }^{d}, n=84\end{array}$ & $\begin{array}{l}\text { Intermediate annual } \\
\text { income }^{e}, n=127\end{array}$ & $\begin{array}{l}\text { High annual } \\
\text { income }{ }^{f}, n=32\end{array}$ & $p$ value \\
\hline FDep & $-0.6[-2 ;-0.1]$ & $-0.6[-2 ; 0]$ & $-0.6[-1.8 ;-0.1]$ & $-1.1[-2.1 ; 0.1]$ & 0.701 \\
\hline FDep $\leq 0$, nondeprived, no. (\%) & $184(75.7 \%)$ & $63(75 \%)$ & $98(77.2 \%)$ & $23(71.9 \%)$ & 0.808 \\
\hline FDep >0, deprived, no. (\%) & $59(24.3 \%)$ & $21(25 \%)$ & $29(22.8 \%)$ & $9(28.1 \%)$ & 0.808 \\
\hline MCS 12 months* & $60.3[36 ; 77.9]$ & $46.2[32.7 ; 65.2]$ & $64.7[40 ; 78]$ & $77.6[63.1 ; 85.6]$ & $<0.001$ \\
\hline PCS 12 months* & $58.3[33.1 ; 80.6]$ & $44.4[28.1 ; 73.1]$ & $58.1[34.8 ; 80]$ & $83.1[56.9 ; 90]$ & 0.001 \\
\hline ICU length of stay & $12[6,19]$ & $9[5 ; 15.2]$ & $13[7 ; 21.5]$ & $16[8,22]$ & 0.011 \\
\hline Hospital length of stay & $23[14 ; 38.5]$ & $20[12.8 ; 33.2]$ & $27[16 ; 40.5]$ & $24[14.5 ; 32.5]$ & 0.06 \\
\hline
\end{tabular}

FDep French Deprivation Index, ICU intensive care unit, MCS mental component scale, PCS physical component scale

*Within 1 year of ICU discharge

a Primary school: "enseignement primaire"', niveau college"

b Undergraduate/high school: "enseignement technique court", "niveau lycée"

c Postgraduate: "1er, 2e, 3e cycle de l'enseignement"

d $<8000-12,000 €$

e $12,000-50,000 €$

f $>50,000-\geq 100,000 €$ 
cutoffs have been reported, thus rendering it rather difficult to find true differences.

\section{Limitations of the study}

We acknowledge that this study has several limitations. First, the observational nature of the data and the lack of a control group hindered confirmation of causality and specific interactions of socioeconomic status on the outcomes. Second, we had no data on several variables that have a major impact on the reported results (i.e., patient compliance and overall behavior [e.g., a readiness to assume risk] and socioeconomic status after hospital discharge), some of which could be compared to the status prior to ICU admission. Additionally, the causes of death after ICU discharge are not known in the FROG-ICU cohort. Third, for all of the presented scores, a baseline status would provide further valuable information for the interpretation of our results. Fourth, instead of comparing our results with the normal French population, it would be interesting to compare the baseline status of hospitalized patients in general and of intensive care patients in particular. Fifth, the unverified answers to the social questionnaire and the rather low percentage of completed overall questionnaires could have biased the results. Sixth, the FDep has not been validated in critical care settings to date. Seventh, our study mostly included hospitals from urban areas and not from "rural" or "quasi-rural" areas (fewer than 10,000 inhabitants); therefore, an aggregation bias could be assumed. As previously described by other authors, it is not fully clear if the gradient described by the FDep truly represents the differences between rural and urban areas. For example, when the FEDI or the Townsend index was used, opposite gradients were observed with deprivation when related to the degree of urbanicity [17]. Therefore, our study did not have a primary focus on explaining the gradient between the urban and rural areas; rather, this study assessed the association between socioeconomic status (using FDep) and outcomes. However, being aware of the aggregational bias and possible gradient between the rural and urbanized geographical regions, we excluded the Paris cohort, as described for the crosschecking analysis. Eighth, the entire study population belonged to the same country, which makes it difficult to extrapolate the results to ICUs in other countries and with other ethnicities. However, the findings of this "real-world" European registry are easier to translate to the general ICU population. Additionally, not all of these limitations are major issues, and the results of our study are unexpected and warrant further exploration in clinical research settings.

\section{Conclusions}

The present study showed no association between 1-year mortality and social deprivation evaluated by the French Deprivation Index. However, long-term physical recovery appeared to be poorer in the socially deprived ICU survivors. In addition, prolonged psychiatric symptoms are common and affect an already vulnerable and slowly recovering patient group. Hence, interventions that occur after ICU discharge should target physical function. Moreover, regular postdischarge screenings for anxiety, depression, or PTSD-like symptoms, as well as an outpatient program with a low threshold for appointments, should be provided for ICU patients.

As critical illness survivors still have more physical and psychological impairments, a lower quality of life, and higher mortality rates than the general population, additional barriers that are created by socioeconomic status should be further investigated, although the best index for determining the differences in health after ICU care remains to be determined. Whether the French Deprivation Index is able to represent the true socioeconomic status has to be further evaluated in a larger cohort of patients, either through the use of the social questionnaire or a similar tool.

\section{Electronic supplementary material \\ The online version of this article (https://doi.org/10.1007/s00134-018-5412-5) contains supplementary material, which is available to authorized users.}

\section{Author details \\ 1 Department of Anaesthesiology and Reanimation, Burn and Critical Care Medicine, AP-HP, Saint Louis and Lariboisière University Hospitals, 2 Rue Ambroise Paré, 75010 Paris, France. ${ }^{2}$ INSERM UMR-S942, Institut National de la Santé et de la Recherche Médicale (INSERM), Lariboisière Hospital and INI- CRCT Network, Paris, France. ${ }^{3}$ Department of Anaesthesiology, Surgical Intensive Care, Prehospital Emergency Medicine and Pain Therapy, University Hospital Basel, Spitalstrasse 21, 4031 Basel, Switzerland. ${ }^{4}$ Université Paris Diderot-Paris 7, Sorbonne Paris Cité, Paris, France. ${ }^{5}$ INSERM UMR1 123, Paris, France. ${ }^{6}$ Unité de Recherche Clinique en Économie de la Santé d'lle-de- France, Paris, France. ${ }^{7}$ Service de Réanimation Médicale, Hôpital Saint-Antoine, Assistance Publique-Hôpitaux de Paris, Université Pierre et Marie Curie, Paris, France. ${ }^{8}$ Department of Anesthesiology and Intensive Care, Bichat University Hospital, Assistance Publique-Hôpitaux de Paris, Paris, France. ${ }^{9}$ Department of Anesthesiology and Intensive Care Medicine, Besançon University Hospital, Besançon, France. ${ }^{10}$ EA 3920, University of Bourgogne Franche-Comté, Besan- çon, France. ${ }^{11}$ Department of Intensive Care Medicine and Infectious Diseases, Université Paris Diderot, Sorbonne Paris Cité, Assistance Publique-Hôpitaux de Paris, Hôpital Bichat-Claude, Paris, France.}

\section{Authors' contributions}

$\mathrm{KB}, \mathrm{EG}, \mathrm{AH}, \mathrm{AM}, \mathrm{MS}$ : Study concept and design. EG, AM: Acquisition of data. KB, $E F, E G, A H, A M, M S$ : Analysis and interpretation of data. $K B, A H$ : Drafting of the manuscript. EF: Critical revision of the manuscript for important intellectual content: all declared authors. EG, AM: Statistical analysis Obtained funding. EG, AM: Administrative, technical, or material support. EG, AM: Study supervision. All authors read and approved the final manuscript.

\section{Funding}

FROG ICU (ClinicalTrials.gov Identifier NCT01367093) was funded by the Programme Hospitalier de la Recherche Clinique (AON 10-216) and by a research grant from the Société Française d'Anesthésie-Réanimation. 


\section{Compliance with ethical standards}

\section{Ethical approval and consent to participate}

The study was conducted in France and Belgium in accordance with Good Clinical Practice (Declaration of Helsinki 2002) and ethics committee approvals (Comité de Protection des Personnes - Ile de France IV, IRB n00003835 and Commission d'éthique biomédicale hospitalo-facultaire de l'hôpital de Louvain, IRB nB403201213352). It is registered on ClinicalTrials.gov (NCT01367093). Patients were enrolled from August 2011 to June 2013.

\section{Consent for publication}

Consent for study participation was obtained from all included patients.

\section{Availability of supporting data}

Prof. Gayat and Prof. Mebazaa had full access to all data in the study and take responsibility for the integrity of the data and the accuracy of the data analysis

\section{Conflicts of interest}

None of the authors of this paper has a financial or personal relationship with other persons or organizations that could inappropriately influence or bias the content of the paper. Etienne Gayat received a research grant from sphingotec and consultancy fees from Magnisense and Roche Diagnostics. Alexandre Mebazaa received speaker's honoraria from Abbott, Novartis, Orion, Roche, and Servier and a fee as a member of the advisory board and/or steering committee from Cardiorentis, Adrenomed, MyCartis, Neurotronik, and sphingotec. Martin Siegemund received speaker's honoraria from Fresenius, Orion Pharma, and SwissOrpha and a research grant from Octapharma. The original FROG ICU study was supported by grants from Assistance Publique-Hôpitaux de Paris (AOR01004) and from Société Française d'Anesthésie-Réanimation.

Received: 30 July 2018 Accepted: 10 October 2018

Published online: 23 October 2018

\section{References}

1. Iwashyna TJ, Ely EW, Smith DM, Langa KM (2010) Long-term cognitive impairment and functional disability among survivors of severe sepsis. JAMA 304:1787-1794

2. Bienvenu OJ, Friedman LA, Colantuoni E, Dinglas VD, Sepulveda KA, Mendez-Tellez P, Shanholz C, Pronovost PJ, Needham DM (2018) Psychiatric symptoms after acute respiratory distress syndrome: a 5-year longitudinal study. Intensive Care Med 44:38-47

3. Moitra VK, Guerra C, Linde-Zwirble WT, Wunsch H (2016) Relationship between ICU length of stay and long-term mortality for elderly ICU survivors. Crit Care Med 44:655-662

4. Graf JJ, Uwe J (2003) Der post-intensivpatient. Langzeitüberleben und lebensqualität nach intensivtherapie. Intensivmed Notfallmedizin 40:184-194

5. Niskanen M, Kari A, Halonen P (1996) Five-year survival after intensive care-comparison of 12,180 patients with the general population. Finnish ICU study group. Crit Care Med 24:1962-1967

6. Riva M, Gauvin L, Barnett TA (2007) Toward the next generation of research into small area effects on health: a synthesis of multilevel investigations published since July 1998. J Epidemiol Community Health 61:853-861

7. Dowdy DW, Eid MP, Sedrakyan A, Mendez-Tellez PA, Pronovost PJ, Herridge MS, Needham DM (2005) Quality of life in adult survivors of critical illness: a systematic review of the literature. Intensive Care Med 31:611-620

8. Alter DA, Franklin B, Ko DT, Austin PC, Lee DS, Oh PI, Stukel TA, Tu JV (2014) Socioeconomic status, functional recovery, and long-term mortality among patients surviving acute myocardial infarction. PLoS One 8:e65130

9. Benaïs M, Da Silva D, Ferreira L et al (2018) Socioeconomic status features of ICU patients: the PRECAREA pilot study. Intensive Care Med. https:// doi.org/10.1007/s00134-018-5380-9

10. Adler NE, Ostrove JM (1999) Socioeconomic status and health: what we know and what we don't. Ann NY Acad Sci 896:3-15
11. Mebazaa A, Casadio MC, Azoulay E, Guidet B, Jaber S, Levy B, Payen D, Vicaut E, Resche-Rigon M, Gayat E (2015) Post-ICU discharge and outcome: rationale and methods of the The [sic] French and euRopean Outcome reGistry in Intensive Care Units (FROG-ICU) observational study. BMC Anesthesiol 15:143

12. Brazier JE, Harper R, Jones NM, O'Cathain A, Thomas KJ, Usherwood T, Westlake L (1992) Validating the SF-36 health survey questionnaire: new outcome measure for primary care. BMJ 305:160-164

13. Rash CJ, Coffey SF, Baschnagel JS, Drobes DJ, Saladin ME (2008) Psychometric properties of the IES-R in traumatized substance dependent individuals with and without PTSD. Addict Behav 33:1039-1047

14. Murphy D, Ross J, Ashwick R, Armour C, Busuttil W (2017) Exploring optimum cut-off scores to screen for probable posttraumatic stress disorder within a sample of UK treatment-seeking veterans. Eur J psychotraumatol 8:1398001

15. Morina N, Ehring T, Priebe S (2013) Diagnostic utility of the impact of event scale-revised in two samples of survivors of war. PLoS One 8:e83916

16. Bjelland I, Dahl AA, Haug TT, Neckelmann D (2002) The validity of the Hospital Anxiety and Depression Scale. An updated literature review. Psychosom Res 52:69-77

17. Temam S, Varraso R, Pornet C, Sanchez M, Affret A, Jacquemin B, ClavelChapelon F, Rey G, Rican S, Le Moual N (2017) Ability of ecological deprivation indices to measure social inequalities in a French cohort. BMC Public Health 17:956

18. Obidoa CA, Reisine SL, Cheniack M (2010) How does the SF-36 perform in healthy populations? A structured review of longitudinal studies. JSBHS 4:30-48

19. Nesseler N, Defontaine A, Launey Y, Morcet J, Malledant Y, Seguin P (2013) Long-term mortality and quality of life after septic shock: a follow-up observational study. Intensive Care Med 39:881-888

20. Geyer S, Peter R (2000) Income, occupational position, qualification and health inequalities - competing risks? (comparing indicators of social status). J Epidemiol Community Health 54:299-305

21. Bouchardy C, Verkooijen HM, Fioretta G (2006) Social class is an important and independent prognostic factor of breast cancer mortality. Int J Cancer 119:1145-1151

22. Storm L, Schnegelsberg A, Mackenhauer J, Andersen LW, Jessen MK, Kirkegaard H (2018) Socioeconomic status and risk of intensive care unit admission with sepsis. Acta Anaesthesiol Scand 62:983-992

23. Blecha S, Schlitt HJ, Graf BM, Leitzmann M, Bein T (2018) Influence of social characteristics on the duration of treatment, severity of the disease and social support of patients in a surgical intensive care unit. Anaesthesist 67:336-342

24. Galiatsatos P, Brigham EP, Pietri J, Littleton K, Hwang S, Grant MC, Hanse NN, Chen ES (2018) The effect of community socioeconomic status on sepsis-attributable mortality. J Crit Care 46:129-133

25. Rush B, Wiskar K, Celi LA, Walley KR, Russell JA, McDermid RC, Boyd JH (2018) Association of household income level and in-hospital mortality in patients with sepsis: a nationwide retrospective cohort analysis. J Intensive Care Med 33:551-6

26. Bradley CJ, Given CW, Roberts C (2001) Disparities in cancer diagnosis and survival Cancer 91:178-188

27. Laffey JG, Madotto F, Bellani G, Pham T, Fan E, Brochard L, Amin P, Arabi Y, Bajwa EK, Bruhn A, Cerny V, Clarkson K, Heunks L, Kurahashi K, Laake JH, Lorente JA, McNamee L, Nin N, Palo JE, Piquilloud L, Qiu H, Jimenez JIS, Esteban A, McAuley DF, van Haren F, Ranieri M, Rubenfeld G, Wrigge H, Slutsky AS, Pesenti A, Investigators LS, Group ET (2017) Geo-economic variations in epidemiology, patterns of care, and outcomes in patients with acute respiratory distress syndrome: insights from the LUNG SAFE prospective cohort study. Lancet Respir Med 5:627-638

28. Gabriel LE, Bailey MJ, Bellomo R, Stow P, Orford N, McGain F, Santamaria J, Scheinkestel C, Pilcher DV (2016) Insurance status and mortality in critically ill patients. Crit Care Resusc 18:43-49

29. OECD iLibrary (2017) Health at a glance. https://doi.org/10.1787/healt h glance-2017-en

30. Parker AM, Sricharoenchai T, Raparla S, Schneck KW, Bienvenu OJ, Needham DM (2015) Posttraumatic stress disorder in critical illness survivors: a metaanalysis. Crit Care Med 43:1121-1129 